\title{
Detailed SiO proper motion analysis: slow net expansion and a small correlation with the magnetic field
}

K. Assaf ${ }^{a d}$, P.J. Diamond ${ }^{b}$, W.D. Cotton ${ }^{c}$, S. Etoka ${ }^{d}$ M.D. Gray ${ }^{d}$, E.M.L. Humphreys ${ }^{e}$, A.M.S. Richards ${ }^{* d}$ and M. Wittkowski ${ }^{e}$

${ }^{a}$ Dept. of Physics, College of Science, University of Wasit, Iraq

${ }^{b}$ SKA Organisation, Jodrell Bank Macclesfield, Cheshire, SK11 9DL, UK

${ }^{c}$ National Radio Astronomy Observatory, 520 Edgemont Road, Charlottesville, VA 22903, USA

${ }^{d} J B C A$, School of Physics and Astronomy, University of Manchester, Manchester M13 9PL, UK

${ }^{e}$ ESO, Karl-Schwarzschild-Str. 2, 85748 Garching bei Munchen, Germany

E-mail: kassaf@uowasit.edu.iq

A detailed analysis of 23 epochs (covering two stellar cycles) of monitoring $\mathrm{R}$ Cas $\mathrm{SiO} \mathrm{J}=1-0$ $\mathrm{v}=1$ masers showed 184 maser features (an average of $20 \%$ per epoch) could be matched over between 3 and 13 epochs. The largest number of matches occur in the early part of each cycle, following the maser brightness trends, which are roughly as predicted. The proper motions can be tangential, radial, intermediate or change direction, but overall the net direction is expansion corresponding to $0.4 \pm 0.1 \mathrm{~km} \mathrm{~s}^{-1}$. This would take $\sim 67$ year to cross the $\mathrm{SiO}$ maser shell, giving a mass loss rate similar to other estimates in the literature. A small proportion of feature pairs in successive epochs have significant polarization and polarization angles consistent to within $\pi / 8$ rad. A small excess of this subsample have proper motion vectors within $22.5 \mathrm{deg}$ of parallel to the inferred magnetic field direction ( $2 \sigma$ significance) but this is in radial expansion for less than half of these. The magnetic field strength provides a force comparable to the kinetic and thermal energy densities and could influence their directions, but it does not appear to be driving the majority of motions of gas clumps. The $\mathrm{SiO}$ masers typically emanate from a shell within $2-5$ optical stellar radii, which is also the region where the radio photosphere becomes optically thick at $\lambda 1.3-6 \mathrm{~cm}$, and where molecular or dust emission can be traced by IR interferometry. Recent advances in techniques and e-MERLIN K-band observations will allow improved coordination between maser and continuum/IR observations in the investigation of how exactly mass is lost from stars.

14th European VLBI Network Symposium \& Users Meeting (EVN 2018)

8-11 October 2018

Granada, Spain

${ }^{*}$ Speaker. 


\section{R Cas, its $\mathrm{SiO}$ masers and mass loss}

R Cassiopeia is a M-type Mira variable star, i.e. an oxygen-rich asymptotic red giant (AGB) star. Its apparent magnitude varies from +4.7 to +13.5 within a time period of 430 days. Its mass is roughly 1.2 solar masses [T98] and its optical photospheric radius $R_{\star}$ is 12.6 mas [W00], 2.22 au at a distance of about $176_{-45}^{+92} \mathrm{pc}$ [V03]. It has a velocity $V_{\star}=24 \pm 2 \mathrm{~km} \mathrm{~s}^{-1}$ (see references in [A11]. All observed velocities are given as the the line of sight velocity with respect to the local standard of rest, $V_{\mathrm{LSR}}$. This paper is based on observations of the $\mathrm{SiO} v=1, J=1-0$ maser transition at 43.11749 GHz made using the VLBA in 1999 - 2001 for 23 useful epochs, spanning stellar phases $\phi 0.158-1.782$. More details and initial results for total intensity were described in [A11] and the polarization properties were introduced in [A13]. The polarization angle calibration is accurate to $\sim 10 \%$ and the fractional linear polarization to $\sim 5 \%$. A movie of the $\mathrm{SiO}$ masers was presented in [A12] and these proper motion results are described in [A13]. Please see these papers for more details.

Here, we analyse the maser proper motions quantitatively in order to tackle the question of whether their trajectories are predominantly ballistic [M08] or are influenced by the magnetic field [K09]. These authors analysed a selection of clumps in IK Tau and TX Cam, respectively. The former traced outflow and infall, attributed to the piston of stellar pulsation followed by fall-back under gravity, the latter found that some features followed non-radial trajectories which the polarization vectors showed were aligned with magnetic field lines.

This work is the first published attempt to trace all maser clumps which can be identified in multiple epochs, to look for any systematic effects which give a clue to what drives mass loss. This is an open question for O-rich AGB stars. Outside $\sim 5 R_{\star}$, there is ample evidence for radiation pressure on dust driving a radially accelerating wind (e.g. review [H18]). Closer to the star, sufficiently absorbent grains would be destroyed by heating; scattering might play a role but requires special grain composition [H16]. It has been suggested that mass loss and dust formation might be enhanced above convection cells, that the magnetic field can shape if not drive the wind [V05], and the correlations between $\mathrm{SiO}$ maser velocity, intensity and stellar pulsations [G09] show that the associated shocks are still significant, although damped in this region.

\section{Maser properties and polarization}

Figure 1 shows the distribution of $\mathrm{SiO}$ masers. We assume that the ring-like appearance is due to tangential beaming from maser clumps which are rapidly accelerating in outflow or infall, such that the velocity-coherent path lengths are greater through the limbs of a spherical shell, than along the line of sight to the star. The plots show the positions and velocities of 2D Gaussian components fitted to maser emission in each channel (of full width half maximum, FWHM $s$, corresponding to the beamed size of the maser emission). These form series in velocity and position, across $\sim 1$ or a few $\mathrm{km} \mathrm{s}^{-1}$ and mas, which correspond to the emitting clouds, total size $L$, feature FWHM $d_{\mathrm{F}}$. Some features show similarities for several epochs, both in spatial and velocity distribution and in polarization angle. We identified 26-69 features per epoch, a total of 938.

We estimated the beaming angle $\delta \Omega=\left(d_{\mathrm{F}} / L\right)^{2}$ and used $s$ to estimate the brightness temperature $T_{\mathrm{B}}$. The mean $\delta \Omega \sim 0.047 \mathrm{sr}$ and the mean $T_{\mathrm{B}} \sim 2.8 \times 10^{9} \mathrm{~K}$. According to [K09], assuming 

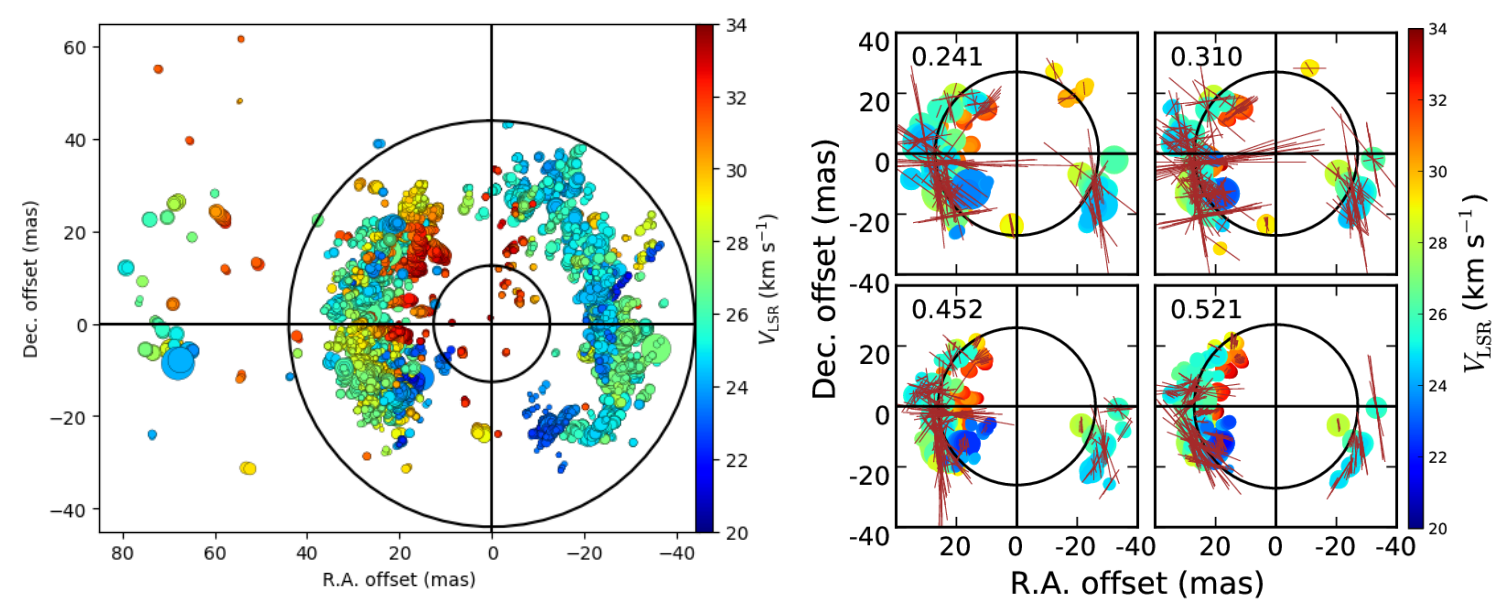

Figure 1: left All 23 epochs $\mathrm{SiO}$ maser spots superimposed, size proportional to $\log$ (flux density). The centre of expansion, assumed to be the stellar centre, is at $(0,0)$. The black circles bound the main ring, the inner limit being $R_{\star}$ and the outer limit, the maximum radius of emission at 21 epochs (7.744 au) (the remaining two have a distinct, outer, partial shell). right Example of 4 epochs (labelled by stellar phase) shown separately with EVPA superimposed, $1 \mathrm{Jy}=5$ mas. Here, the ring shows the average $\mathrm{SiO}$ ring radius per epoch.

that these masers are mostly saturated, the stimulated emission rate is given by:

$$
R=23 \frac{T_{\mathrm{B}}}{2 \times 10^{10} \mathrm{~K}} \times \frac{\delta \Omega}{0.01 \mathrm{sr}} \mathrm{s}^{-1}
$$

Here, $R \sim 15 \mathrm{~s}^{-1}$. We were unable to measure circular polarization accurately but single-dish measurements of the $86 \mathrm{GHz} \mathrm{SiO}$ masers, coming from a similar region [H06], gave a field strength of 0.9-2.8 G. This leads to a Zeeman splitting rate $g \Omega 900 \mathrm{~s}^{-1}$ [P03]. The radiative decay rate for the transition we observed at $43 \mathrm{GHz} \Gamma<1 \mathrm{~s}^{-1}$, so $g \Omega \gg R \gg \Gamma$ which supports a magnetic origin of maser polarization [G73].

This means that the maser electric vector polarization angle (EVPA) is either parallel or perpendicular to the magnetic field in the same plane, since when the the angle $\theta$ between the magnetic field direction and the direction of maser propagation (i.e. the line of sight) is $>55^{\circ}$ (the van Vleck angle) the EVPA are perpendicular to the magnetic field lines; for $\theta<55^{\circ}$ they are parallel [E92]; [G73]. We observe such a change of direction in a few maser features. For a radial magnetic field, this suggests that the ring has a depth in the plane of the sky with an opening angle allowing just a few features to cross this limit, corresponding to an angle $\sim 50^{\circ}$ from the line of sight. Spherical trigonometry then leads to the assumption that very roughly, $64 \%$ of the clumps are visible in $\mathrm{SiO}$ masers.

\section{SiO maser proper motions and mass loss rate}

We matched features detected in successive epochs within their angular and velocity extents, with an allowance for error (mainly due to uncertainty in aligning epochs), i.e. within $\sim 3$ mas in $x$ and $y$ and $2 \mathrm{~km} \mathrm{~s}^{-1}$. We matched a total of 184 features in successive epochs. Less than $40 \%$ 
of features in any epoch could be matched, an average of $20 \%$ over all epochs. Epochs at phases $\phi 0.31-0.45$ and 1.03 had the most matched features $(\geq 15)$; there were $\leq 5$ matched features for $\phi 0.68-82$ and $\phi \geq 1.23$, with zero in the final epoch at $\phi 1.78$. This mostly reflects the maser flux density variation with phase (and thus detectability and position accuracy). We only consider series of features identified over 3 or more epochs; the longest series spans 13 epochs. It is likely that the physical features survive longer than the conditions for masing, as at least one series fades and re-appears (19-20). The matched features are shown in Fig. 2; all are in the main ring.
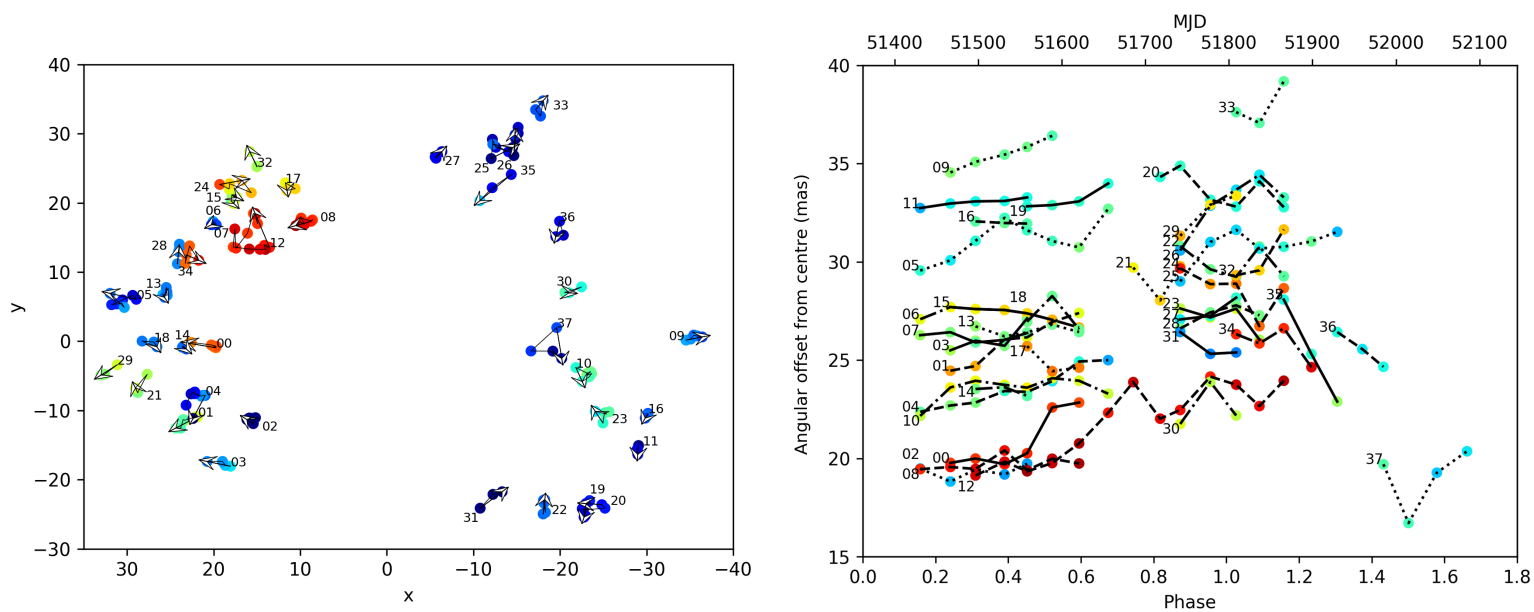

Figure 2: left Matched $\mathrm{SiO}$ maser features, the arrow shows their overall direction of motion. right The radial trajectories of matched features as a function of time or stellar phase.

The maser Doppler velocities with respect to $V_{\star}$ are up to $\sim \pm 7 \mathrm{~km} \mathrm{~s}^{-1}$; since we do not see the front and back caps properly the maximum $\left|V_{\mathrm{LSR}}-V_{\star}\right| \sim 10 \mathrm{~km} \mathrm{~s}^{-1}$. Owing to uncertainties, the maximum allowed proper motion is somewhat larger. However, the mean radial proper motion velocity over all epochs was $+0.4 \pm 0.1 \mathrm{~km} \mathrm{~s}^{-1}$ away from the star. There was no significant, systematic rotation. Ireland [I11] derived mass loss models based on stellar parameters, which we scaled to the properties of $\mathrm{R}$ Cas. Using the temperatures and number densities within a spherical shell corresponding to the main ring, and the mean proper motion, we derive a mass loss rate of $(4 \pm 1) \times 10^{-7} \mathrm{M}_{\odot} \mathrm{yr}^{-1}$. The mass loss rate of $\mathrm{R}$ Cas has been estimated from observations of dust etc. as $\sim 6 \times 10^{-7} \mathrm{M}_{\odot} \mathrm{yr}^{-1}$ [W01] or $\sim 1 \times 10^{-6} \mathrm{M}_{\odot} \mathrm{yr}^{-1}$ [T98] (by methods independent from the assumptions used in [I11]). Given the uncertainties, this is a good agreement and suggests that the net outward $\mathrm{SiO}$ maser motion over $\sim 2$ cycles is representative of the rate of mass loss.

\section{Proper motions and magnetic field direction.}

We extended the discussion in Section 3 to estimate the mass in the SiO maser clumps. Assuming that the clumps survive even when not masing, we took the most populous epochs as typical and included the $\sim 24 \%$ of the clumps not beaming masers in our direction (end of Section 2). The average clump extent is $0.3 \mathrm{au}$, mass $\sim 2.6 \times 10^{-9} \mathrm{M}_{\odot}$, giving a filling factor $\leq 1 \%$ (similar to that of $22 \mathrm{GHz} \mathrm{H}_{2} \mathrm{O}$ maser clumps further out in the wind [R12]) and average total mass in clumps per 
epoch $\sim 1.4 \times 10^{-7} \mathrm{M}_{\odot}$. We could then estimate the mean energy density of each cloud from thermal and bulk motions, $\sim 0.016 \mathrm{~J} \mathrm{~m}^{-3}$, and the equivalent magnetic energy density, which would require a mean field strength of $2 \mathrm{G}$ (range $0.2-4.5 \mathrm{G}$ ). This is comparable to the range of 0.9-2.8 $\mathrm{G}$ measured by [H06]. Thus, the magnetic field is strong enough to influence the outflow direction, but is there any alignment between the proper motion direction and the field lines?

We took each pair of matched features and selected those where both had significant polarization measurements and the EVPA differed by less than either $11^{\circ} .25$ (or $22^{\circ} .5$ ) (being slightly less or more than the EVPA uncertainty). This gave 13 (or 22) pairs. Within these, we compared the EVPA alignment with the direction of proper motion, in $45^{\circ}$ bins of difference in these angles (Fig. 3, left). The bin covering orthogonal proper motion and EVPA directions is the most popular. If we assume that the magnetic field is predominantly radial and the masers come from close to the plane of the sky, this implies a $2 \sigma$ preference for the magnetic field and the direction of proper motion being close to aligned (for either cut-off in EVPA alignment accuracy).
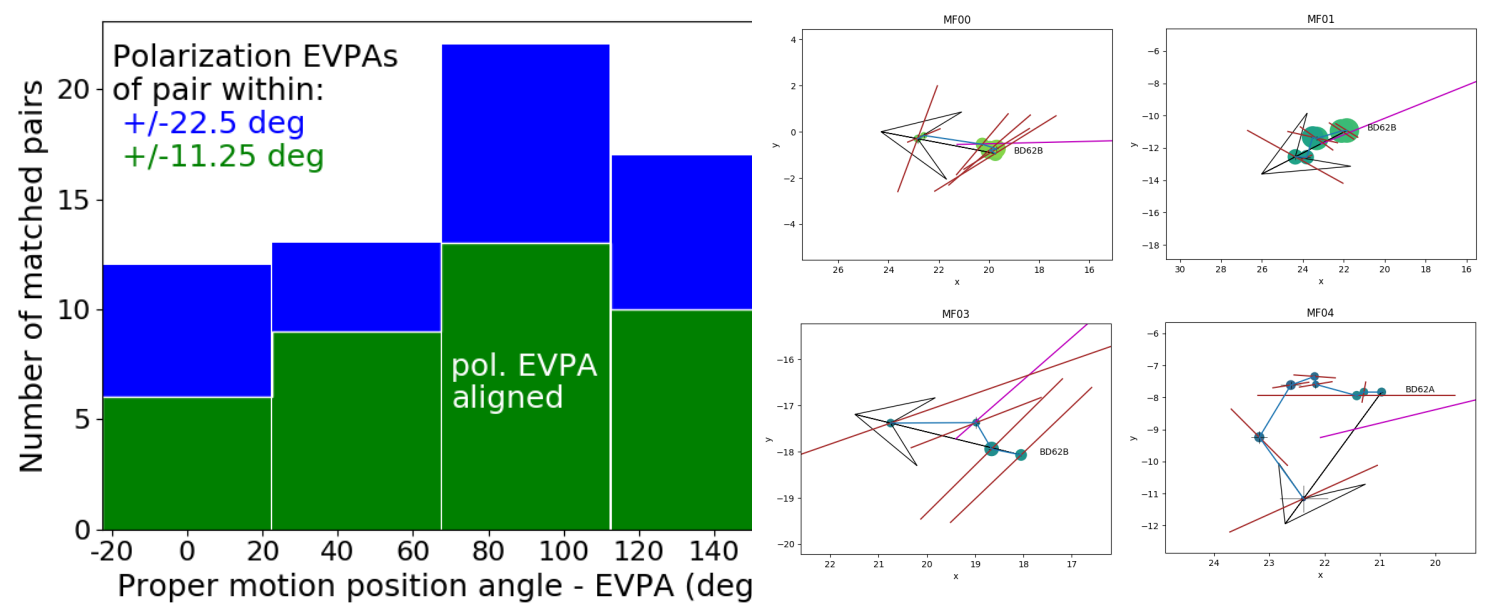

Figure 3: left The distribution of the difference between the proper motion vector and the average EVPA, for pairs of features with EVPA differing by $11^{\circ} .25$ (green) or $22^{\circ} .5$ (blue). right Examples of matched $\mathrm{SiO}$ maser features, the arrow shows their overall direction of motion. The magenta line shows the direction of the star and the brown lines, the EVPA. In the top row the proper motions are approximately radial but the bottom row shows tangential and tangled motions.

However, only about half of these pairs come from series with predominantly radial proper motions, of which only 4 are consistently in expansion. Fig. 3 (right) shows examples of series with different behaviour.

\section{Conclusions}

About $20 \%$ of $\mathrm{SiO}$ maser features can be identified continuously for 3-13 epochs out of 23 . The masing clumps probably survive longer but the conditions for masing vary [G09] The wind takes 50-70 years to cross the $\mathrm{SiO}$ maser shell at a net expansion speed of $\sim 0.4 \mathrm{~km} \mathrm{~s}^{-1}$ (less than a tenth of the individual clump velocities, which are however in many directions). Such a long $\mathrm{SiO}$ shell crossing time could help dust formation. We used the model of Ireland and Gray, based on stellar properties, to deduce the mass density in the $\mathrm{SiO}$ maser shell, and combined with 
the net expansion, deduced a mass loss rate $(4 \pm 1) \times 10^{-7} \mathrm{M}_{\odot} \mathrm{yr}^{-1}$, within $50 \%$ of independent measurements in the literature.

There is a slight preference for an alignment between the magnetic field and proper motion directions, and, within that, for radial expansion. However, the great majority of features do not show such alignments, but have complex field and proper motion directions. This is partly due to intrinsic complexity in the wind, and partly to the difficulties (at the time of observations) in securing accurate polarization calibration, as well as some epochs being affected by bad weather. Future observations could take advantage of advances in linear and circular polarization calibration. Phase referencing would allow model-independent negligent of epochs, or, better still, sufficient sensitivity to spatial scales of 5-50 mas to detect the star (typically 1 or a few mJy for nearby Miras at $43 \mathrm{GHz}$ ). Moreover, between 10 and $90 \%$ of the flux of different features is resolved-out, adding uncertainty to the estimates of clump size and beaming angle. Short spacings $(<100$ to a few $100 \mathrm{~km}$ ) would help this also.

\section{References}

[A11] Assaf K. A., Diamond P. J., Richards A. M. S. and Gray M. D., 2011, MNRAS, 415, 1083.

[A12] Assaf K. A.,2012,PhD thesis, (Silicon monoxide masers and the magnetic field of R Cassiopeiae) https://www.research.manchester.ac.uk/portal/files/54520074/FULL_TEXT.PDF

[A13] Assaf K. A., Diamond P. J., Richards A. M. S. and Gray M. D., 2013, MNRAS., 431, 1077.

[A13] Assaf K. A., 2019, ApJ, accepted.

[E92] Elitzur, M., 2002, Astronomical Masers, Dordrecht: Kluwer

[G73] Goldreich, P., Keeley, D. A. and Kwan, J. Y., 1973, ApJ., 179, 111.

[G09] Gray M. D., Wittkowski, M., Scholz, M., et al., 2009, MNRAS, 394, 51.

[H06] Herpin, F., Baudry, A., Thum, C., Morris, D. and Wiesemeyer, H. 2006, A\&A, 450, 667.

[H16] Höfner, S., 2016, A\&A, 594, 108.

[H18] Höfner, S., 2016 and Olofsson, H., 2018, A\&ARv, 26, 1.

[I11] Ireland, M. J., Scholz, M. and Wood, P. R., 2011, MNRAS, 418, 114.

[K09] Kemball A. J., Diamond P. J., Gonidakis I., et al., 2009, ApJ., 698, 1721.

[M08] Matsumoto, N. et al., 2008, PASJ, 60, 1039.

[P03] Plambeck, R. L., Wright, M. C. H. and Rao, R. 2003, ApJ. 594, 911.

[R12] Richards, A. M. S., Etoka, S., Gray, M. D., et al., 2012, A\&A, 516, 16.

[T98] Truong-Bach, Nguyen-Q-Rieu, Sylvester, R. J., et al., 1998, Ap\&SS, 255, 325.

[V03] Vlemmings W. H. T., van Langevelde H. J., Diamond P. J., Habing H. J. and Schilizzi R. T., 2003, A\&A, 407, 213.

[V05] Vlemmings W. H. T., van Langevelde H. J. and Diamond P. J., 2005, A\&A, 434, 1029.

[W00] Weigelt G. et al. 2000, in Léna P., Quirrenbach A., eds, Proc. SPIE Vol. 4006, Interferometry in Optical Astronomy. SPIE, Bellingham, p. 617.

[W01] Weigelt, G. and Yudin, B. F., 2001, ARep, 45, 510. 